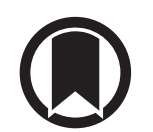

CrossMark

\section{High nicotine exposure in rodents is unlikely to inform about its toxicity in humans}

To the Editor:

We read with interest the research letter by ReINIKOVAITE et al. [1], in which the authors reported same level of damage in the lungs of Sprague Dawley rats subjected to whole body chronic exposure of e-cigarette vapour emissions and cigarette smoke, as well as to subcutaneous injection of nicotine. Their conclusion is that e-cigarette use and long-time consumption of nicotine are just as toxic as tobacco cigarettes. We appreciate the authors' intention to address concerns related to the potential long-term health effects of e-cigarette use and nicotine exposure, but there are a number of methodological considerations that lessen the impact of their findings.

First, in spite of the authors' effort to establish a meaningful puff/puff comparison between e-cigarette vapour and cigarette smoke, rats weighting about $200 \mathrm{~g}$ were being exposed to 600 puffs of vapour or smoke in just over $4 \mathrm{~h}$ in small gas chambers of $<1 \mathrm{~m}^{3}$ volume. Humans weigh, on average, 400 times more than Sprague Dawley rats and yet they usually inhale the same 600 puffs in just over 3 days. Under these specific experimental conditions, animals' overexposure to aerosol mass and its contents is highly likely. This is not uncommon, as there is proliferation of toxicological studies that are often performed with high-dose exposure protocols that are not applicable to normal human consumption [2]. When calculating the dose to be used, particularly if this is based upon human data, allometric scaling should be used, to take into account the large differences in metabolic rate between animals of different body mass [3, 4]. Moreover, in the work of Reinikovaite et al. [1], rats were exposed daily for $4 \mathrm{~h}$ for a total of 5 weeks; such exposure protocol is designed to maximise harm for the induction of pathological status (e.g. emphysema or chronic obstructive pulmonary disease (COPD)), which is used to study the characteristics of the disease in animal models (inflammation, basic mechanisms, response to therapies, etc.) [5]. Also, we noted that the authors did not mention animal health during the study, and standard toxic endpoints detailed in Organisation for Economic Cooperation and Development test number 413 (organ weights, gross pathology, etc.) were not assessed [6].

Second, in trying to equate similar level of plasma nicotine of adult smokers in the much smaller animal model (weighing approximately 400 times less than humans), the authors end up injecting a highly toxic dose. In histopathological studies of rats, it has been found that subcutaneous administration of nicotine doses similar to those used by ReInikovaite et al. [1] can cause extensive tissue damage, not only in the lung, but also in other organs including the liver, kidneys and brain [7, 8]. Thus, the dose used in the study is toxic and far from being informative of the nicotine effects in humans under the normal condition of consumption. It would have been ideal to include a rat group exposed to e-cigarette without nicotine to reach more revealing conclusions. Moreover, given that it is well established that the protease/ anti-protease ratio is the central component of our understanding of emphysema, it is incongruous to advance the alternative hypothesis that it is nicotine causing lung destruction in smokers. This hypothesis is also at variance with the epidemiological evidence; for example, biomass fuel combustion is a well-known causative factor for COPD not dependent on nicotine $[9,10]$.

Third, in stark contrast with the concerns raised by ReINIKOVAITE et al. [1] that e-cigarettes are as toxic as tobacco cigarettes and can cause lung damage, there is emerging evidence from real-life surveys [11] and the prospective evaluation of lung function and high-resolution computed tomography of regular vapers

@ERSpublications

More realistic exposures protocols are required to provide reliable toxicological information about electronic cigarette use in the context of smoking harm reduction http://bit.ly/2YkZHWG

Cite this article as: Emma R, Polosa R, Caruso M. High nicotine exposure in rodents is unlikely to inform about its toxicity in humans. Eur Respir J 2019; 54: 1801073 [https://doi.org/10.1183/13993003.010732018]. 
who have never smoked in their life [12], indicating that long term e-cigarette use is unlikely to raise significant lung health concerns. Moreover, despite their being millions of regular e-cigarette users worldwide, there has been no evidence of emerging respiratory disease outbreaks in recent years.

Rosalia Emma $\oplus^{1}$, Riccardo Polosa ${ }^{1,2}$ and Massimo Caruso $\odot^{1}$

${ }^{1}$ Dept of Clinical and Experimental Medicine, University of Catania, Catania, Italy. ${ }^{2}$ Center of Excellence for the acceleration of HArm Reduction (CoEHAR), University of Catania, Catania, Italy.

Correspondence: Massimo Caruso, University of Catania, Dept of Clinical and Experimental Medicine, Hospital University, Via S. Sofia, 78, Catania, CT 95125, Italy. E-mail: mascaru@unict.it

Received: June 082018 | Accepted after revision: June 162019

Conflict of interest: R. Emma has no conflicts of interest to disclose. R. Polosa has served as a consultant for Pfizer, Global Health Alliance for treatment of tobacco dependence, ECITA (Electronic Cigarette Industry Trade Association, in the UK), Arbi Group Srl, and Health Diplomats (a consulting company that delivers solutions to global health problems with special emphasis on harm minimisation); has received lecture fees from a number of European electronic cigarette industry and trade associations (including FIVAPE in France and FIESEL in Italy) which were directly donated to vapers advocacy non-profit organisations; and is also currently involved in the following pro bono activities: scientific advisor for LIAF, Lega Italiana Anti Fumo (Italian acronym for Italian Anti-Smoking League), the Consumer Advocates for Smoke-free Alternatives (CASAA) and the International Network of Nicotine Consumers Organizations (INNCO); chair of the European Technical Committee for standardisation on "Requirements and test methods for emissions of electronic cigarettes" (CEN/TC 437; WG4). M. Caruso has no conflicts of interest to disclose.

\section{References}

1 Reinikovaite V, Rodriguez IE, Karoor V, et al. The effects of electronic cigarette vapour on the lung: direct comparison to tobacco smoke. Eur Respir J 2018; 51: 1701661.

2 Volti G L, Polosa R, Caruso M. Assessment of E-cigarette impact on smokers: the importance of experimental conditions relevant to human consumption. Proc Natl Acad Sci 2018; 115: E3073-E3074.

3 Nair AB, Jacob S. A simple practice guide for dose conversion between animals and human. J Basic Clin Pharm 2016; 7: 27-31.

4 Huang Q, Riviere JE. The application of allometric scaling principles to predict pharmacokinetic parameters across species. Expert Opin Drug Metab Toxicol 2014; 10: 1241-1253.

5 Ghorani V, Boskabady MH, Khazdair MR, et al. Experimental animal models for COPD: a methodological review. Tob Induc Dis BioMed Central 2017; 15: 25.

6 Organisation for Economic Cooperation and Development. OECD Guidelines for the Testing of Chemicals, Section 4. Test No. 413: Subchronic Inhalation Toxicity: 90-day Study. https://doi.org/10.1787/9789264070806-en Date last accessed: February 11, 2019.

7 Adluri RS, Nagarajan D, Periyaswamy V, et al. Dose-response effect of ferulic acid against nicotine-induced tissue damage and altered lipid levels in experimental rats: a pathohistological evaluation. Fundam Clin Pharmacol 2008; 22: 557-567.

8 Balakrishnan A, Menon VP. Effect of hesperidin on nicotine toxicity and histopathological studies. Toxicol Mech Methods 2007; 17: 233-239.

9 Kurmi OP, Semple S, Simkhada P, et al. COPD and chronic bronchitis risk of indoor air pollution from solid fuel: a systematic review and meta-analysis. Thorax 2010; 65: 221-228.

10 Salvi SS, Barnes PJ. Chronic obstructive pulmonary disease in non-smokers. Lancet 2009; 374: 733-743.

11 Farsalinos KE, Romagna G, Tsiapras D, et al. Characteristics, perceived side effects and benefits of electronic cigarette use: a worldwide survey of more than 19,000 consumers. Int J Environ Res Public Health 2014; 11: 4356-4373.

12 Polosa R, Cibella F, Caponnetto P, et al. Health impact of E-cigarettes: a prospective 3.5-year study of regular daily users who have never smoked. Sci Rep 2017; 7: 13825.

\section{Lessons learned from animal models: adverse effects of electronic cigarettes}

From the author:

We thank R. Emma and colleagues for their interest in our preclinical study to investigate the effects of e-cigarettes on the lung. Our study was initiated from clinical observations that children exposed to second-hand e-cigarette vapour at home and undergoing surgery have a longer recovery and healing time 
than children from a non-smoking environment. For this reason, our animals underwent whole body (or so called second-hand smoke) exposure to e-cigarette vapour.

We must clarify that the whole body exposure is very different from direct inhalation and the number of puffs delivered into the $32-\mathrm{m}^{3}$ exposure chamber in experimental conditions were comparable to the use of electronic cigarettes and tobacco cigarettes by people.

The amount of nicotine used for injections was based on previous studies to produce stable plasma nicotine levels of approximately $25 \mathrm{ng} \cdot \mathrm{mL}^{-1}$, which is comparable with plasma levels (in the $10-$ $50 \mathrm{ng} \cdot \mathrm{mL}^{-1}$ range) of habitual smokers $[1,2]$. In our study nicotine was injected as nicotine di-tartrate salt. Thus, the actual dose used was below high toxicity levels [2] and we believe that our data are highly informative of the nicotine effects in humans under normal conditions of consumption.

We closely monitored animal health during the 35-day exposure period and, as anticipated, animals exposed to cigarette smoke lost close to $15 \%$ of the body weight when compared to room air exposed controls. The same body weight loss was observed in animals exposed to e-cigarette vapour or subjected to nicotine injections. We documented the adverse effects of e-cigarette exposure by lung pathology [3] and by skin necrosis in a skin flap survival model in vivo [4]. In a skin flap survival model, we showed that exposure to e-cigarette vapour produced damaging effect on skin microcirculation and interfered with the healing process. Our findings suggest that e-cigarette exposure is harmful not only to the lung, but also to the skin microcirculation.

We believe that our study on the adverse effects of e-cigarettes merely touches on the tip of the iceberg. New emerging studies in animal models and cell cultures show that flavourings alone used in e-juices can trigger an inflammatory response in monocytes, mediated by ROS production, endothelial dysfunction, DNA damage and induction of apoptosis in endothelial and epithelial cells. These studies provide insights into potential pulmonary toxicity and tissue damage in e-cigarette users [5-10].

It is premature to draw longitudinal conclusions from a prospective 3.5-year study of non-smoking daily e-cigarette users [11]. More studies are urgently needed regarding the public health consequences of e-cigarettes; so far, the scientific body of evidence on the health effects of e-cigarettes is very limited [12, 13]. Alarmingly, e-cigarette use from 2017 to 2018 increased 78\% among high school students (11.7\% to 20.8\%) and $48 \%$ among middle school students (3.3\% to 4.9\%) from 2017 to 2018 [14], and poisoning events leading to emergency room visits related to liquid nicotine in electronic nicotine delivery systems in young children have been on the rise [15].

We strongly believe that considering recent discoveries the regulatory policy recommendations for humans based on preclinical studies are highly valuable. Due to the much longer life span, humans experience toxic effects after decades of smoking, while preclinical animal models, due to a much shorter time scale, usually demonstrate the first warning signs of possible adverse effects of e-cigarette vapour to human health.

Our data show that e-cigarettes have a signature of harm in the lung that challenges the concept that switching from cigarettes to e-cigarettes is a healthier alternative.

@ERSpublications

Electronic cigarettes have a signature of harm in the lung that challenges the concept that switching from cigarettes to e-cigarettes is a healthier alternative http://bit.ly/2Z3Ahdu

Cite this article as: Taraseviciene-Stewart L. Lessons learned from animal models: adverse effects of electronic cigarettes. Eur Respir J 2019; 54: 1901338 [https://doi.org/10.1183/13993003.01338-2019].

Laimute Taraseviciene-Stewart

Dept of Medicine, Division of Pulmonary and Critical Care Sciences, University of Colorado, School of Medicine, Aurora, CO, USA.

Correspondence: Laimute Taraseviciene-Stewart, Dept of Medicine, Division of Pulmonary and Critical Care Sciences, University of Colorado, Denver, 12700 E. 19th Ave, Aurora, CO, 80045, USA. E-mail: laima.taraseviciene@ucdenver.edu

Received: July 182019 | Accepted: July 182019

Conflict of interest: L. Taraseviciene-Stewart has nothing to disclose.

\section{References}

1 Benwell ME, Balfour DJ, Birrell CE. Desensitization of the nicotine-induced mesolimbic dopamine responses during constant infusion with nicotine. Br J Pharmacol 1995; 114: 454-460.

2 Matta SG, Balfour DJ, Benowitz NL, et al. Guidelines on nicotine dose selection for in vivo research. Psychopharmacology 2007; 190: 269-319. 
3 Reinikovaite V, Rodriguez IE, Karoor V, et al. The effects of electronic cigarette vapour on the lung: direct comparison to tobacco smoke. Eur Respir J 2018; 51: 1701661.

4 Rau AS, Reinikovaite V, Schmidt EP, et al. Electronic cigarettes are as toxic to skin flap survival as tobacco cigarettes. Ann Plast Surg 2017; 79: 86-91.

5 Muthumalage $\mathrm{T}$, Prinz $\mathrm{M}$, Ansah $\mathrm{KO}$, et al. Inflammatory and oxidative responses induced by exposure to commonly used e-cigarette flavoring chemicals and flavored e-liquids without nicotine. Front Physiol 2017; 8: 1130 .

6 Lee HW, Park SH, Weng MW, et al. E-cigarette smoke damages DNA and reduces repair activity in mouse lung, heart, and bladder as well as in human lung and bladder cells. Proc Natl Acad Sci USA 2018; 115: E1560-E1569.

7 Lerner CA, Sundar IK, Yao H, et al. Vapors produced by electronic cigarettes and e-juices with flavorings induce toxicity, oxidative stress, and inflammatory response in lung epithelial cells and in mouse lung. PLoS One 2015; 10: $\mathrm{e} 0116732$.

8 Reidel B, Radicioni G, Clapp P, et al. E-Cigarette use causes a unique innate immune response in the lung involving increased neutrophilic activation and altered mucin secretion. Am J Respir Crit Care Med 2018; 197: 492-501.

9 Ahmad S, Zafar I, Mariappan N, et al. Acute pulmonary effects of aerosolized nicotine. Am J Physiol Lung Cell Mol Physiol 2019; 316: L94-L104.

10 Chun LF, Moazed F, Calfee CS, et al. Pulmonary toxicity of e-cigarettes. Am J Physiol Lung Cell Mol Physiol 2017; 313: L193-L206.

11 Polosa R, Cibella F, Caponnetto P, et al. Health impact of E-cigarettes: a prospective 3.5-year study of regular daily users who have never smoked. Sci Rep 2017; 7: 13825.

12 Eaton DL, Kwan LY, Stratton K, eds. Public Health Consequences of E-Cigarettes. National Academies of Sciences, Engineering, and Medicine; Health and Medicine Division; Board on Population Health and Public Health Practice; Committee on the Review of the Health Effects of Electronic Nicotine Delivery Systems. Washington, National Academies Press (US), 2018.

13 Farsalinos K. Electronic cigarettes: an aid in smoking cessation, or a new health hazard? Ther Adv Respir Dis 2018; 12: 1753465817744960 .

14 Cullen KA, Ambrose BK, Gentzke AS, et al. Notes from the field: increase in use of electronic cigarettes and any tobacco product among middle and high school students - United States, 2011-2018. MMWR Morbid Mortal Wkly Rep 2018; 67: 1276-1277.

15 Chang JT, Wang B, Chang CM, et al. National estimates of poisoning events related to liquid nicotine in young children treated in US hospital emergency departments, 2013-2017. Int Epidemiol 2019; 6: 10.

Copyright @ERS 2019 